Departamento de Ciência e Tecnologia, Secretaria de Ciência, Tecnologia e Insumos Estratégicos, Ministério da Saúde

\section{Rede Nacional de Pesquisa Clínica do Brasil: respostas e redução da dependência estrangeira}

\author{
National clinical research network: \\ Brazilian response and mitigation of \\ international dependency
}

Correspondência | Correspondence:

Decit - Departamento de Ciência e Tecnologia

do Ministério da Saúde

Esplanada dos Ministérios

Bloco G sala 845

70058-900 Brasília, DF, Brasil

Texto de difusão técnico-científica do Ministério de Saúde. a desenvolver ferramentas próprias com a capacidade de solucionar desafios específicos do País.

A construção de uma rede equipada e adequada para a pesquisa, com grupos de investigadores bem treinados, com capacidade para conduzir, compreender e aplicar os resultados das pesquisas, permitirá à RNPC participar de fortalecimento e desenvolvimento tecnológicos no complexo industrial da saúde. O desenvolvimento da tecnologia nacional, com capacidade para competir com a tecnologia estrangeira, constitui também uma meta da RNPC.

os ministérios da Saúde (MS) e da Ciência e Tecnologia (MCT), foi lançado, em abril de 2005, um edital para oferecer apoio financeiro à criação ou consolidação de centros de pesquisa clínica vinculados a hospitais universitários.

Dessa chamada pública foram selecionados 19 centros, formando a Rede Nacional de Pesquisa Clínica em Hospitais de Ensino (RNPC). Foram destinados R \$ 35 milhões para aquisição de equipamentos básicos e infraestrutura necessários ao desenvolvimento de atividades de pesquisa, bem como para a remuneração das equipes por meio de bolsas de pesquisa. Todos os centros são vinculados a instituições de ensino, contemplando todas as regiões brasileiras, visando incorporar as vocações regionais de pesquisa às práticas de fomento, dentro da perspectiva de gestão descentralizada.

A RNPC foi idealizada com o objetivo de incentivar a integração dos centros de pesquisa clínica para proporcionar maior intercâmbio entre pesquisadores e incrementar a produção científica e tecnológica em todo o território nacional, reunindo esforços em ações prioritárias para a população brasileira. Além disso, com a capacitação regional proveniente da integração nacional dirigida, haverá aceleração do crescimento e capacidade científica de cada um desses centros, que estarão aptos

\section{Por que instituir uma rede nacional de pesquisa clínica?}

Atualmente, há inúmeros protocolos de pesquisa clínica em andamento no País. A grande maioria refere-se às prioridades dos laboratórios contratantes, não dedicando espaço para as prioridades do SUS. A participação dos pesquisadores brasileiros, habitualmente, restringese em executar protocolos desenvolvidos em outros países, estando a análise e a apropriação dos dados integralmente no âmbito das firmas contratantes.

O estabelecimento de uma rede de centros de pesquisa clínica contribui para suprir as necessidades de estruturação de espaço físico e de recursos humanos bem treinados, colocando o Brasil em uma situação de maior autonomia quanto ao desenvolvimento de seus estudos clínicos estratégicos. No momento de afirmação da política industrial e de incentivo à produção nacional de medicamentos, faz-se oportuno implantar e assegurar esse tipo de cenário aos pesquisadores brasileiros.

Ainda, o estímulo à inovação deve se embasar na maior interação entre os serviços de saúde, as instituições de ensino e de pesquisa e o complexo produtivo, via parceria ética com as indústrias. Sem uma base produtiva 
qualificada e relativamente desenvolvida, o conhecimento gerado na área da saúde tende a ficar restrito ao âmbito acadêmico ou a ser apropriado economicamente pelas empresas dos países desenvolvidos. A situação de dependência produtiva e tecnológica coloca sério entrave estrutural à expansão das ações de saúde, podendo comprometer os princípios de universalidade, eqüidade e integralidade do SUS. O fortalecimento dos centros de pesquisa e tecnologia das instituições de ensino superior diminuirá a dependência nacional no campo tecnológico e produtivo e garantirá auto-suficiência nos itens estratégicos para o País.

A construção do componente tecnológico da política de pesquisa em saúde deve ser realizada com base em evidências concretas da situação de mercado, das possibilidades tecnológicas locais e, principalmente, das necessidades nacionais com ênfase na realização de estudos que melhor orientem os gestores públicos e privados envolvidos.

\section{O Decit e a RNPC}

Após a implantação da RNPC, em 2005, foram desenvolvidas ações para estreitar a colaboração entre os centros e definir aspectos técnico-operacionais. Assim, em 2006, foi instituído o Comitê Supervisor Técnico, composto por: um representante do Departamento de Ciência e Tecnologia (Decit) do Ministério da Saúde, que o coordena; um representante da Financiadora de Estudos e Projetos (Finep) do Ministério da Ciência e Tecnologia; um representante do CNPq; e o coordenador de cada uma das unidades que integram a rede.

Juntamente com a estruturação física, a maioria dos centros iniciou o processo de treinamento, com a realização de cursos de capacitação e de boas práticas em pesquisa clínica.

A estratégia governamental reúne os parceiros necessários na construção de um novo modelo de pesquisa clínica, voltado às reais necessidades do SUS, visando à consolidação dessa Rede para, prioritariamente, padronizar e executar todas as fases de ensaios clínicos de fármacos, procedimentos, equipamentos e dispositivos para diagnósticos.

Em 2007, os esforços foram concentrados na realização de uma primeira oficina, que definiu as prioridades de estudo na área de Pesquisas Clínicas, com o objetivo de selecionar as tecnologias e temas candidatos a estudos, por meio de chamada pública. As condições de saúde prioritárias eleitas foram diabetes, doenças cardiovasculares, obesidade e doenças negligenciadas.

Naquele ano, a partir dos temas sugeridos na oficina, os ministérios da Saúde e da Ciência e Tecnologia, por intermédio da Finep, publicaram a chamada pública
MCT/Finep/MS/SCTIE/Decit - CT-SAÚDE e FNS - para Pesquisa Clínica 02/2007. Essa chamada comprometeu R $\$ 10$ milhões como recursos não reembolsáveis, sendo R $\$ 4$ milhões oriundos do FNDCT/Fundo Setorial de Saúde e R 6 milhões do Fundo Nacional de Saúde/Decit/SCTIE/MS. Dos recursos financeiros concedidos, $30 \%$ foi destinado às regiões Norte, Nordeste e Centro-Oeste. Nove projetos foram selecionados: três de avaliação da cirurgia bariátrica no Brasil; dois sobre insulinas recombinantes análogas à humana; e quatro sobre o tratamento da leishmaniose.

Em 2008, repetiram-se os esforços para priorizar temas relevantes ao SUS no que se refere à pesquisa clínica, em parceria com as áreas técnicas do Ministério da Saúde, agências reguladoras (Agência Nacional de Vigilância Sanitária - Anvisa e Agência Nacional de Saúde Suplementar-ANS), gestores da saúde e pesquisadores. Uma nova oficina de prioridades foi realizada e, a partir dela, foi elaborada e publicada a chamada pública MCTFinep/MS/SCTIE/Decit - FNS e CT-Saúde - Pesquisa Clínica 02/2008, com previsão de contratação de oito projetos e recurso de $\mathrm{R} \$ 20$ milhões. Dessas propostas, quatro dedicaram-se a avaliar questões relacionadas à apnéia do sono, uma dedicou-se à osteoporose, outra à prevenção de eventos cardiovasculares em pacientes hipertensos, e outras duas à hanseníase.

Em função da relevância e do volume de ações relacionadas à pesquisa clínica coordenada pelo Decit, foi criada, no segundo semestre de 2009, a Coordenação Geral de Pesquisa Clínica (CGPC). Entre outras atribuições, compete a essa coordenação identificar, fomentar, avaliar e monitorar projetos de pesquisa clínica, bem como incentivar e fortalecer o trabalho colaborativo dos centros de pesquisa, respeitando as suas especificidades regionais. Cabe à CGPC também promover e articular cooperação técnico-científica das redes existentes com outras instituições voltadas à pesquisa clínica, sejam elas nacionais, sejam internacionais.

Outra ação importante conduzida pelo Decit, em 2009, foi a ampliação da RNPC, conforme estabelecido no Programa Mais Saúde. ${ }^{\mathrm{b}}$

Considerando a extensão geográfica do território brasileiro, foi necessário ampliar o número de centros de pesquisa na RNPC. A proposta de ampliação da RNPC consistiu nas seguintes etapas: a) estabelecer os critérios de elegibilidade, definidos conjuntamente pelo Decit, RNPC e Finep; b) fazer busca ativa dos centros com potencial para cumprir os critérios estabelecidos; c) elaborar termo de adesão às instituições elegíveis e carta convite para o diretor do hospital das instituições de ensino; e) homologar resultado em cerimônia conjunta com os ministros da Saúde e da Ciência e Tecnologia e os coordenadores da RNPC.

\footnotetext{
b Ministério da Saúde. Secretaria Executiva. Mais saúde: direito de todos: 2008-2001. 2. Ed. Brasília, DF; 2008.
} 
Dessa forma, o Decit realizou um levantamento dos centros de pesquisa, elaborou os critérios para ingresso na RNCP e o termo de adesão, discutidos e aprovados pela Finep e pelo centro coordenador da RNCP.

Os critérios aprovados, listados a seguir, correspondem aos itens descritos na chamada pública que originou a RNPC em 2005:

1. ser hospital vinculado a instituição de ensino, pública ou privada, sem fins lucrativos;

2. ser hospital de ensino com projetos de pesquisa clínica anteriormente selecionados nos editais do Decit, em parceria com o Conselho Nacional de Desenvolvimento Científico e Tecnológico (CNPq) ou com a Finep;

3. ter disponibilidade de instalações físicas em ambiente de enfermarias ou de ambulatórios dos respectivos hospitais para realização de pesquisa clínica;

4. ter certificação como hospital de ensino emitido pelo MS e MEC, de acordo com a portaria interministerial $n^{\circ}$. 1.000, de 15/04/2004;

5. dispor de uma equipe composta, no mínimo, por um coordenador técnico, um enfermeiro com treinamento em pesquisa clínica, um bioestatístico ou um farmacêutico.

6. ter autonomia para o desenvolvimento de pesquisas clínicas formuladas por pesquisadores do próprio hospital e por outros centros, mediante contratos de prestação de serviços;

7. instituir um comitê gestor indicado pelo diretor do hospital, com equipe mínima, constituída por um membro do Comitê de Ética em Pesquisa (CEP) e, no mínimo, mais três pesquisadores clínicos da instituição, com experiência em pesquisa clínica;

8. priorizar o desenvolvimento de protocolos de pesquisa que forem prioritários para as políticas públicas de saúde do País;

9. ter instrumentos de gestão de suas atividades visando autonomia financeira;

10. apresentar coordenador do centro de pesquisa cadastrado na Plataforma Lattes.

A ampliação da RNPC contribui para ajustar o papel da pesquisa clínica à sua rota estratégica de desenvolvimento científico, acompanhando avanços tecnológicos obtidos especialmente no âmbito do complexo produtivo da saúde. Desse processo, 13 centros foram considerados aptos a ingressar na fase de ampliação da RNPC.
A cerimônia de ampliação ocorreu em 25 de novembro de 2009 e contou com a participação dos ministros de Ciência e Tecnologia e da Saúde; do secretário de Ciência, Tecnologia e Insumos Estratégicos do MS; da diretora do Decit; da coordenadora da RNPC; da coordenadora geral de Pesquisa Clínica do Decit; e de representantes de todos os centros de pesquisa da RNPC. Todos os centros receberam certificado de integração à RNPC e assinaram o Termo de Adesão. Após a cerimônia de ampliação em 2009, foi realizada reunião, quando foram criados quatro grupos de trabalho: a) capacitação, que visa identificar as necessidades de aperfeiçoamento de recursos humanos; b) regulação, que tem a atribuição de levantar e discutir questões relacionadas à regulamentação da pesquisa clínica, particularmente ao sistema CEP/Conep e à Anvisa; c) institucionalização, que busca fortalecer os centros membros da rede dentro do hospital de ensino, garantindo sua sustentabilidade; d) novas moléculas, que têm por finalidade identificar pesquisas com potencial de inovação no setor saúde.

\section{Desafios}

Os desafios atuais para o fortalecimento da RNPC estão relacionados à sustentabilidade no financiamento e ao gerenciamento dos centros. As experiências internacionais mostram que o financiamento privado é importante e se estabelece com a capacidade de elaboração e execução de projetos das unidades. No entanto, é necessário um contingente de recursos contínuo e renovável, obtidos de forma competitiva, com recursos públicos que atendam a projetos de interesse para o desenvolvimento tecnológico e em consonância com as políticas de saúde.

Outro desafio da Rede são os novos estudos que ela se propõe a fomentar, ou seja, os estudos nacionais, longitudinais, multidisciplinares e translacionais, capazes de gerar respostas aos problemas de saúde da população brasileira.

\section{COMENTÁRIOS}

A implantação dos centros de pesquisa clínica vinculados a hospitais de ensino visou, inicialmente, à disponibilização de uma infra-estrutura básica para o desenvolvimento de todas as fases de ensaios clínicos de fármacos. Isso permite que os investigadores clínicos possam alcançar novos conhecimentos científicos referentes a medicamentos, procedimentos ou métodos de abordagem de problemas que afetam a saúde da população.

A integração das atividades do governo, das universidades e das empresas nos modernos sistemas de ciência, tecnologia e inovação existentes nos países industrializados praticamente eliminou a defasagem entre a 
Tabela. Centros de pesquisa integrantes da Rede Nacional de Pesquisa Clínica em hospitais de ensino e região.

\begin{tabular}{|c|c|}
\hline Instituições Integrantes da Rede antes da Ampliação & Região \\
\hline Hospital Universitário Clementino Fraga Filho da Universidade Federal do Rio de Janeiro & Sudeste \\
\hline Instituto de Medicina Integral Professor Fernandes Figueira & Sudeste \\
\hline Instituto Nacional de Câncer & Sudeste \\
\hline Hospital das Clínicas da Faculdade de Medicina de Ribeirão Preto da Universidade de São Paulo & Sudeste \\
\hline Hospital de Clínicas da Faculdade de Medicina da Universidade de São Paulo & Sudeste \\
\hline Hospital das Clínicas da Faculdade de Medicina de Botucatu da Universidade Estadual Paulista & Sudeste \\
\hline Hospital Universitário Antônio Pedro da Universidade Federal Fluminense & Sudeste \\
\hline Hospital das Clínicas da Universidade Federal de Minas Gerais & Sudeste \\
\hline Hospital São Lucas da Pontifícia Universidade Católica do Rio Grande do Sul & Sul \\
\hline Hospital de Clínicas de Porto Alegre da Universidade Federal do Rio Grande do Sul & Sul \\
\hline Instituto Materno Infantil Professor Fernando Figueira & Nordeste \\
\hline Hospital Universitário Walter Cantídio da Universidade Federal do Ceará & Nordeste \\
\hline Hospital Universitário Oswaldo Cruz e Pronto Socorro Cardiológico de Pernambuco da Universidade de Pernambuco & Nordeste \\
\hline Hospital Universitário Professor Edgard Santos da Universidade Federal da Bahia & Nordeste \\
\hline Hospital Messejana da Universidade Federal do Ceará & Nordeste \\
\hline Hospital Universitário da Universidade Federal do Maranhão & Nordeste \\
\hline Fundação de Medicina Tropical do Amazonas & Norte \\
\hline Hospital Universitário João de Barros Barreto da Universidade Federal do Pará & Norte \\
\hline Hospital das Clínicas da Universidade Federal de Goiás & Centro-Oeste \\
\hline Instituições que integraram a RNPC em sua ampliação & Região \\
\hline Instituto Nacional de Cardiologia de Laranjeiras & Sudeste \\
\hline Instituto Nacional de Traumato-Ortopedia & Sudeste \\
\hline Hospital Universitário da Universidade de São Paulo & Sudeste \\
\hline Hospital São Paulo da Universidade Federal de São Paulo & Sudeste \\
\hline Hospital das Clínicas da Universidade Estadual de Campinas & Sudeste \\
\hline Fundação Oswaldo Cruz & Sudeste \\
\hline Hospital Universitário Regional de Maringá da Universidade Estadual de Maringá & Sul \\
\hline Hospital Nossa Senhora da Conceição da Universidade do Sul de Santa Catarina & Sul \\
\hline Instituto de Cardiologia do Rio Grande do Sul da Fundação Universitária de Cardiologia & Sul \\
\hline Hospital Geral de Fortaleza & Nordeste \\
\hline Hospital São Rafael & Nordeste \\
\hline Hospital Universitário Onofre Lopes da Universidade Federal do Rio Grande do Norte & Nordeste \\
\hline Hospital Universitário de Brasília da Universidade de Brasília & Centro-Oeste \\
\hline
\end{tabular}

descoberta e a aplicação, além de ter criado o círculo virtuoso de retroalimentação positiva entre a pesquisa e o desenvolvimento socioeconômico (Kriegerc 2010 ). Nesse contexto, a RNPC, por meio da pesquisa clínica genuinamente nacional, poderá propiciar capacitação tecnológica, autonomia e auto-suficiência tecnológicas para a construção de uma política tecnológica, competitiva e de inovação no âmbito do complexo industrial.

\footnotetext{
${ }^{c}$ Krieger EM. Perspectivas da medicina translacional. Folha de Sao Paulo, 2010 fev 18; Caderno Opinião: A3.
} 